Article

\title{
Functional Analysis of Maize Silk-Specific ZmbZIP25 Promoter
}

\author{
Wanying Li, Dan Yu, Jingjuan Yu, Dengyun Zhu and Qian Zhao * \\ State Key Laboratory of Agrobiotechnology, College of Biological Sciences, China Agricultural University, \\ No. 2 Yuanmingyuan West Road, Haidian District, Beijing 100193, China; liwanyingcau@foxmail.com (W.L.); \\ danyupw155@yeah.net (D.Y.); yujj@cau.edu.cn (J.Y.); zhudy@cau.edu.cn (D.Z.) \\ * Correspondence: zhaoqian@cau.edu.cn; Tel.: +86-10-6273-3330
}

Received: 8 February 2018; Accepted: 3 March 2018; Published: 12 March 2018

\begin{abstract}
ZmbZIP25 (Zea mays bZIP (basic leucine zipper) transcription factor 25) is a function-unknown protein that belongs to the D group of the bZIP transcription factor family. RNA-seq data showed that the expression of ZmbZIP25 was tissue-specific in maize silks, and this specificity was confirmed by RT-PCR (reverse transcription-polymerase chain reaction). In situ RNA hybridization showed that ZmbZIP25 was expressed exclusively in the xylem of maize silks. A $5^{\prime}$ RACE (rapid amplification of cDNA ends) assay identified an adenine residue as the transcription start site of the ZmbZIP25 gene. To characterize this silk-specific promoter, we isolated and analyzed a $2450 \mathrm{bp}$ (from -2083 to +367) and a $2600 \mathrm{bp}$ sequence of ZmbZIP25 (from -2083 to +517 , the transcription start site was denoted +1 ). Stable expression assays in Arabidopsis showed that the expression of the reporter gene GUS driven by the $2450 \mathrm{bp} \mathrm{ZmbZIP25} 5^{\prime}$-flanking fragment occurred exclusively in the papillae of Arabidopsis stigmas. Furthermore, transient expression assays in maize indicated that GUS and GFP expression driven by the 2450 bp ZmbZIP25 5'-flanking sequences occurred only in maize silks and not in other tissues. However, no GUS or GFP expression

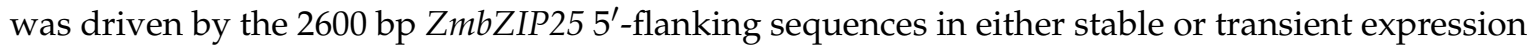
assays. A series of deletion analyses of the $2450 \mathrm{bp} \mathrm{ZmbZIP25} 5^{\prime}$-flanking sequence was performed in transgenic Arabidopsis plants, and probable elements prediction analysis revealed the possible presence of negative regulatory elements within the $161 \mathrm{bp}$ region from -1117 to -957 that were responsible for the specificity of the ZmbZIP25 5'-flanking sequence.
\end{abstract}

Keywords: bZIP transcription factor; intron; maize; silk specificity; $5^{\prime}$-flanking sequence

\section{Introduction}

Silk is a necessary part of the female reproductive organ in maize. Each maize silk is a specialized long trichome anchored to each ovule, which is functionally equivalent to the stigma and style in typical flowering plants [1-3]. Silk is responsible for catching pollen grains and supporting pollen hydration and germination. Pollen grains germinate to form a pollen tube after hydration on the silk; this tube grows parallel to the vascular bundles and reaches the ovule. The nutrition provided by pollen grains support the formation of 2-cm-long pollen tubes [1], while the subsequent nutrition and signal factors needed by the growing pollen tubes are provided by silk. The growth and development of maize silks directly relate to pollination and fertilization. Hairs located on the silk surface serve as receptive structures to aid pollen adhesion, hydration, and germination [4]. To accomplish these physiological processes, maize silks might express genes that are specific or at least preferential to silk.

Several genes that are specifically or preferentially expressed in stigmas have been identified. SSP (stigma-specific peroxidase), isolated from Senecio squalidus L., is expressed in specialized epidermal cells of stigmas. SSP may enhance protection against pathogen attack when the stigma is "primed" to receive pollen $[5,6]$. Other genes that function primarily in the stigma include the stigma-specific $S$ 
locus receptor kinase from tobacco [7], proteinase inhibitors from Nicotiana alata stigmas [8], and genes involved in the self-incompatibility system of Brassica [9-12]. In maize, a transcriptome analysis showed that 1427 genes were specifically or preferentially expressed in silk [4]. Bioinformatic analyses revealed that many of these genes function in plant reproductive systems and that some of these genes encode amino acid transporters, peptide and oligopeptide transporters, and cysteine-rich receptor-like kinases [4]. However, few reports of research on silk-specific promoters exist. Tao et al. cloned a gene named zmgrp5 (Zea mays glycine-rich protein 5), which encodes a 187-amino-acid glycine-rich protein that is expressed specifically in maize silks. Transient expression analyses revealed that this $1779 \mathrm{bp}$ $z m g r p 5$ promoter fused with $\beta$-glucuronidase (GUS) was expressed in silk but not in leaf. Heterologous expression in Arabidopsis showed that a zmgrp5 promoter-GUS fusion was expressed highly in stigmas and at low levels in the filaments and vascular elements of the petals [13].

Silks are one of the main ways in which fungi such as Fusarium graminearum invade maize [14]. Fungal spores grow and germinate on the silks, and hyphae then spread to the outside and inside of the silks until they infect the ovules. Silks are also susceptible to infection by insect pests such as corn earworm (Helicoverpa zea) [15]. The expression of some defense genes that benefit from silk-specific promoters in silk may increase resistance to fungi and pests. A $p 1$ (pericarp1) gene encoding a $M y b$ transcription factor is driven by the putative silk-specific promoter $p S H 64$, enhancing resistance to Helicoverpa zea [16]. Research on silk-specific promoters is also of great value in production. Sterility-related genes can be driven by silk-specific promoters to produce sterile female plants. Female sterility plays a role in genetic breeding and seed production by effectively preventing self-pollination.

In this study, we showed that ZmbZIP25 is expressed specifically in maize silk. Expression analysis of both maize and Arabidopsis showed that the region of ZmbZIP25 from -2083 to +367 contains the promoter activity. A detailed promoter deletion analysis was performed and identified a region from -1117 to -957 that is required for ZmbZIP25 specificity. Moreover, aberrant splicing of the second intron of ZmbZIP25 might lead to the inability to express reporter genes in transgenic Arabidopsis.

\section{Results}

\subsection{ZmbZIP25 Is Silk-Specifically Expressed in Maize}

ZmbZIP25 (Zea mays bZIP (basic leucine zipper) transcription factor 25, GRMZM2G080731) encodes a polypeptide of 346 amino acid residues. ZmbZIP25 belongs to the D group of the bZIP transcription factor family according to its phylogenetic relationship with Arabidopsis and rice proteins [17]. Transcriptome sequencing data from MaizeGDB (28/8/2017, http:/ / www.maizegdb.org) indicate that ZmbZIP25 has high-level expression in silks and little expression in other tissues (Figure 1A). To confirm the expression pattern of ZmbZIP25, we performed an RT-PCR (reverse transcription-polymerase chain reaction) analysis. The results showed that the ZmbZIP25 transcript was present in silk but not in other organs, including root, shoot, leaf, cob, tassel, seed 5 DAP (day after pollination), aerial root, and stem (Figure 1B).

To better understand the expression position of ZmbZIP25 in silks, we performed an in situ hybridization analysis using maize silks during flower development. The hybridization results of both cross sections and longitudinal sections showed that ZmbZIP25 was exclusively expressed in the xylem of dumbbell-like maize silk cells (Figure 2).

The protein structure of ZmbZIP25 was predicted to contain a bZIP domain and a DOG1 (delay of germination 1) domain. DOG1 accumulates during seed maturation and participates in the seed dormancy mechanism of Arabidopsis. In situ hybridization assays have indicated that DOG1 is mainly expressed in the vascular tissues of the embryo [18]. ZmbZIP25 was expressed in the vascular tissues of maize silk, which is consistent with the location of DOG1, but was not expressed in maize seed. This result implies that ZmbZIP25 may not participate in seed dormancy. The group D genes of bZIP participate in two different processes: defense against pathogens and development. TGA (TGACG motif-binding factor) transcription factors bind to the as- 1 cis-element present in the promoters of 
pathogenesis-related (PR) genes and induce the expression of PR genes in response to pathogen attack [17]. Maize silks are vulnerable to fungal invasion and insect feeding. The expression of genes in silk is responsible for resisting fungal invasion and insect feeding during pollination and fertilization in maize. ZmbZIP25 may be involved in regulating PR genes.
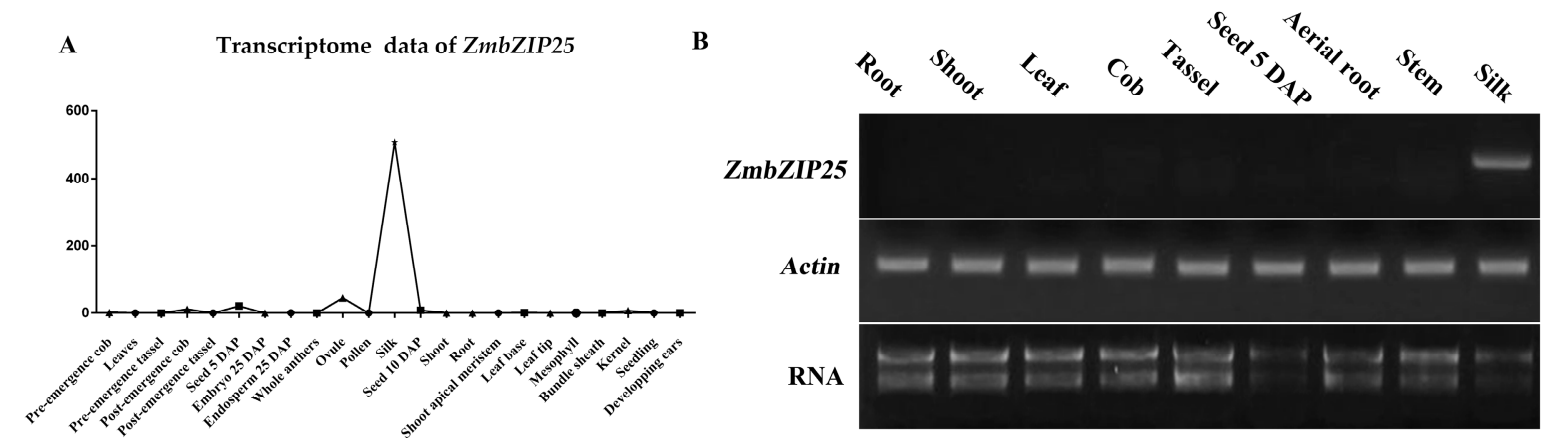

Figure 1. Expression analysis of ZmbZIP25 in maize. (A) Line chart analysis of ZmbZIP25 transcriptome data. The $x$-axis represents tissues at various developmental periods. The $y$-axis represents transcriptome data (transcriptome data from the MaizeGDB). (B) RT-PCR (reverse transcription-polymerase chain reaction) analysis of the expression of the ZmbZIP25 gene in different maize tissues. RNA was extracted from tissues as indicated above. Actin served as a control. DAP, day after pollination.
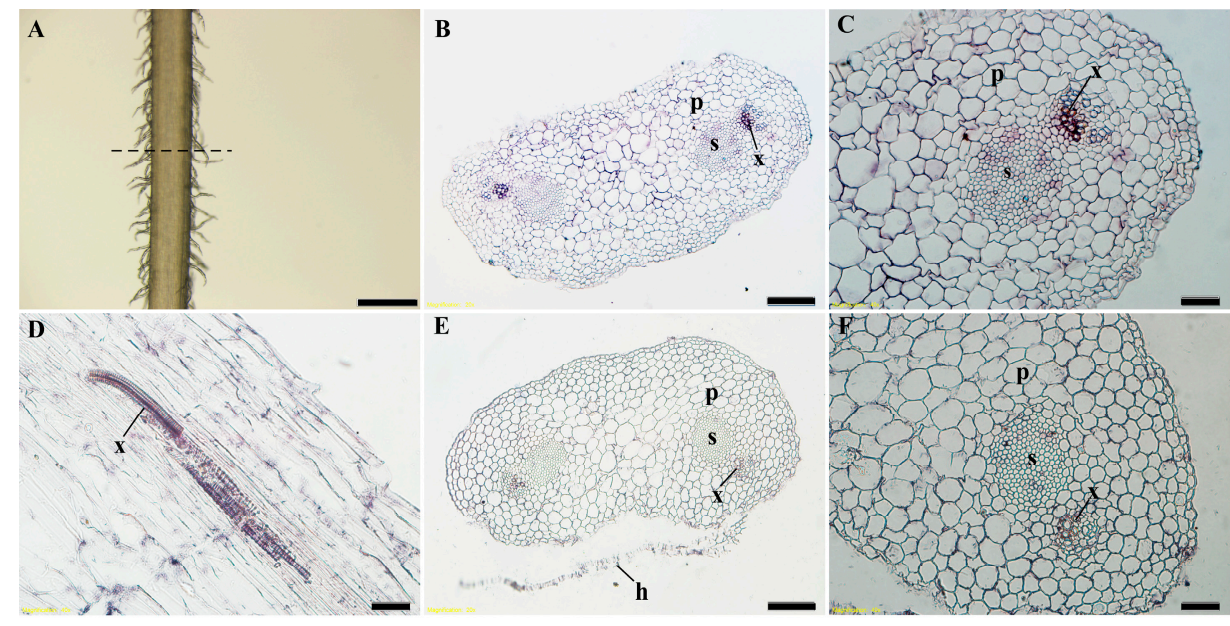

Figure 2. Spatial localization of ZmbZIP25 transcripts in silk. In situ RNA hybridization was performed on silks collected on the day of emergence. (A) Fresh maize silk under a stereo microscope, with the dotted line representing the axis of cross sections. Cross section of a silk strand with antisense probes for ZmbZIP25 (B) and enlarged detail (C). (D) A Sample derived from the longitudinal section of maize silk with antisense probes for ZmbZIP25. (E) Negative control with sense probes for ZmbZIP25 and enlarged detail (F). Abbreviations: p, parenchyma cell; s, sheath cell; $x, x y l e m ; h$, hair. Bars $=500 \mu \mathrm{m}$ in (A), $50 \mu \mathrm{m}$ in (B,E), and $20 \mu \mathrm{m}$ in $(\mathbf{C}, \mathbf{D}, \mathbf{F})$.

\subsection{Analysis of Cis-Regulatory Elements in ZmbZIP25 Upstream Region}

To characterize the regulatory mechanisms controlling the transcription of ZmbZIP25, we analyzed the upstream region of ZmbZIP25. The upstream sequences of ZmbZIP25 were accessed in Phytozome v12 (https:/ / phytozome.jgi.doe.gov/pz/portal.html\#). Surprisingly, a series of Ns was present in the upstream sequences ( -443 to -344 ; predicted transcription start site (TSS) was +1 ) of the putative ZmbZIP25 genomic sequence at the website (Figure S1). In addition, multiple occurrences of these upstream series of Ns were found when BLAST searches were performed. The sequence information 
available online was that of the genetic background of the B73 inbred line. Considering possible errors during sequence splicing, we found the upstream sequences of ZmbZIP25 from the Mo17 inbred line (from unpublished data), which appears correct and lacks the "Ns" of uncertain nucleic acids. The $5^{\prime}$ upstream region of ZmbZIP25 was thus cloned from the Mo17 inbred line.

A $5^{\prime}$ RACE (rapid amplification of cDNA ends) assay was performed to identify the TSS of the ZmbZIP25 gene. Amplified fragments (approximately $500 \mathrm{bp}$ in length) were sequenced to determine the $5^{\prime}$ ends of the products. Sequence analyses showed that the ZmbZIP25 gene contains only one TSS. An adenine residue flanked by cytosine and thymine bases and located $555 \mathrm{bp}$ upstream of the translation start codon initiates gene transcription (Figure 3). This result was consistent with the presence of an adenine in the TSS that is flanked by pyrimidine bases in most plant genes [19]. By comparing cDNA sequences with the genomic sequences of ZmbZIP25, we identified two introns in the region between the TSS and the start codon $(+67 /+160$ and $+285 /+444$; the TSS A was designated +1 ). It is possible that $5^{\prime}$ untranslated regions ( $5^{\prime}$ UTRs) play roles in regulating gene expression, including regulating mRNA stability and translation efficiency [20]. The $2600 \mathrm{bp}$ fragment from -2083 to +517 that contains upstream sequences and a large part of the $5^{\prime}$ UTR was analyzed.
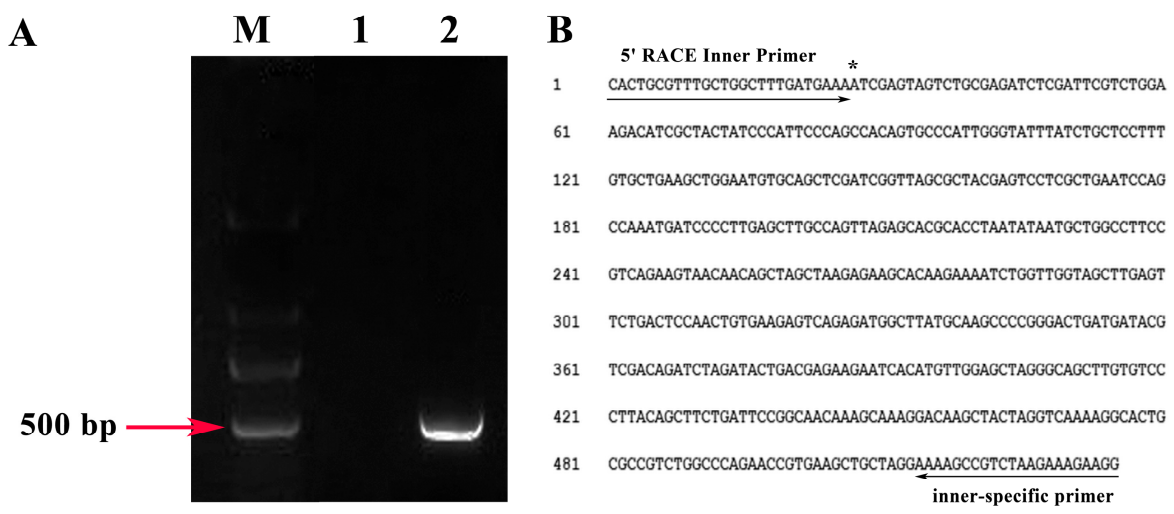

Figure 3. Identification of ZmbZIP25 transcription start site by $5^{\prime}$ RACE assay. (A) $5^{\prime}$ RACE ((rapid amplification of cDNA ends) PCR results. M, DNA marker DL2000 Plus. 1, outer $5^{\prime}$ RACE PCR products. 2, inner $5^{\prime}$ RACE PCR products. (B) Sequence alignment result. The arrows represent the primers. The asterisk "**" represents the transcription start site.

Nine putative TATA boxes (core element of promoters, at $-1215,-1150,-875,-840,-822$, $-781,-769,-725$, and -552 ) and five CAAT boxes (proximal element of promoters, at $-1113,-941$, $-452,-285$, and -42 ) were identified in this $2600 \mathrm{bp}$ fragment using the plant cis-acting regulatory element database (http://bioinformatics.psb.ugent.be/webtools/plantcare/html/) (Figure 4 and Figure S2). Interestingly, the putative TATA box was not within $50 \mathrm{bp}$ upstream of the TSS. Nevertheless, two YTCANTYY initiator elements were found in the ZmbZIP25 $5^{\prime}$-flanking sequence. In addition, some motifs found in the ZmbZIP25 5'-flanking sequence, such as the AGCCGCC motif and the GCC-box (http://bioinfo.cau.edu.cn/ProFITS/), are involved in the promoters of many pathogen-responsive genes, which implies that ZmbZIP25 might be involved in pathogen resistance in maize silk. 


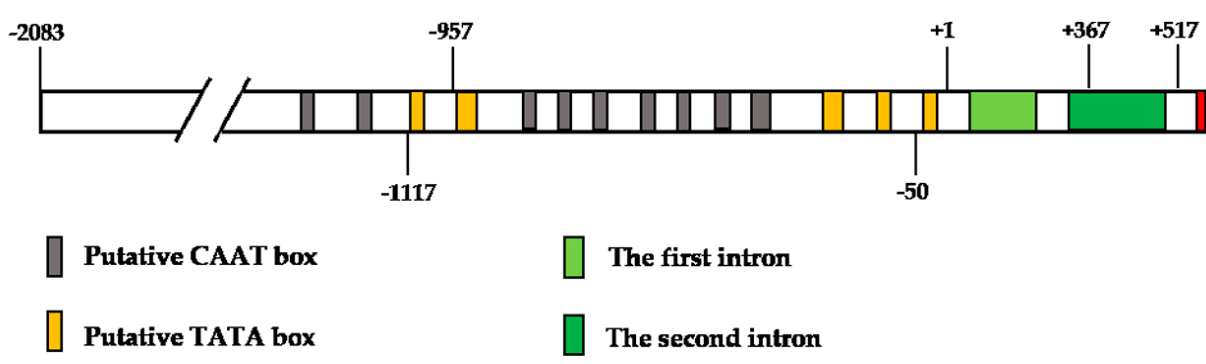

Start codon ATG

Figure 4. The schematic representation of the $5^{\prime}$-flanking region of ZmbZIP25. The transcription start site identified by $5^{\prime}$ RACE was denoted +1 . Putative TATA and CAAT boxes in the promoter region are denoted by gray and yellow blocks, respectively. The start codon is denoted by a red block. The first intron and the second intron are denoted by light-green and green blocks, respectively.

\subsection{Stigmatic Papillae-Specific Transcription in Arabidopsis and the Impact of the Second Intron of ZmbZIP25}

To characterize the $5^{\prime}$-flanking region of ZmbZIP25 in plants, we analyzed a $2600 \mathrm{bp}$ fragment ranging from -2083 to +517 and a $2450 \mathrm{bp}$ fragment ranging from -2083 to +367 . Heterologous transgenic Arabidopsis plants were generated. The plasmids p2600 and p2450 were each transformed into Arabidopsis via the Agrobacterium-mediated floral dip method. Histochemical analysis of $\mathrm{T}_{2}$ generation Arabidopsis plants showed that blue staining occurred specifically in the papillae of Arabidopsis stigmas (Figure 5C) but not in seedling (Figure 5A) or rosette leaf (Figure 5B) when harboring p2450. Surprisingly, no staining was detected in Arabidopsis seedling, rosette leaf, or flower when harboring p2600 (Figure 5D-F). These results indicated that the GUS gene was not expressed from p2600. In addition, the $2450 \mathrm{bp}$ fragment $(-2083 /+367)$ of the ZmbZIP25 5 -flanking region exhibited promoter activity and could specifically drive reporter gene expression.
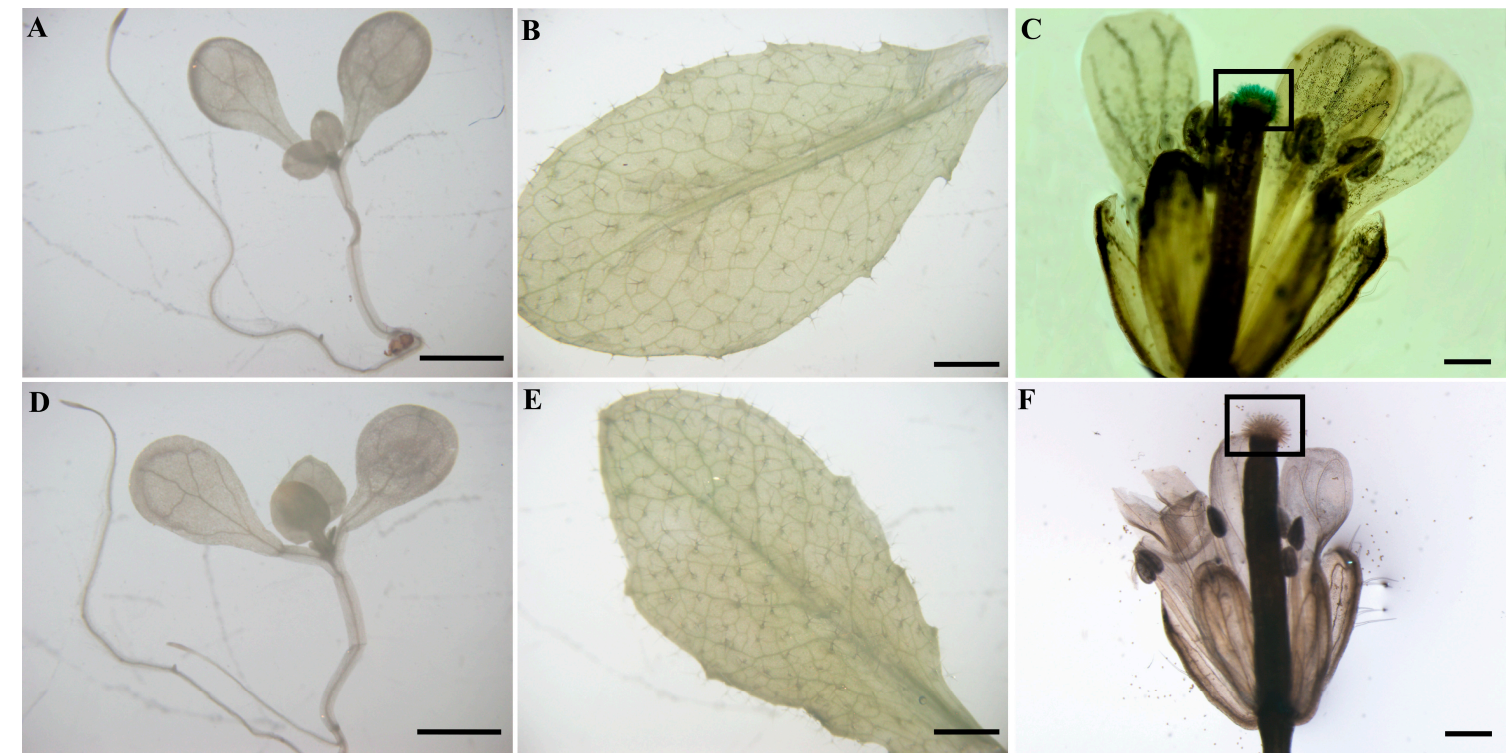

Figure 5. Histochemical analysis of $\beta$-glucuronidase (GUS) activity in Arabidopsis. Histochemical analysis of GUS activity in seedling (A), rosette leaf (B), and flower (C) of $\mathrm{T}_{2}$ transgenic Arabidopsis harboring p2450; histochemical analysis of GUS activity in seedling (D), rosette leaf (E), and flower (F) of $\mathrm{T}_{2}$ transgenic Arabidopsis harboring p2600. The stigmatic papillae are boxed in (C,F). At least six independent transformants were examined for each construct. Bars $=500 \mu \mathrm{m}$ in $(\mathbf{C}, \mathbf{F})$, and $2 \mathrm{~mm}$ in $(\mathbf{A}, \mathbf{B}, \mathbf{D}, \mathbf{E})$. 
The $2450 \mathrm{bp}$ fragment lacked $151 \mathrm{bp}$ at its $3^{\prime}$ end that was present in the $2600 \mathrm{bp}$ fragment. This $151 \mathrm{bp}$ sequence contained a part of the second intron and a part of the $5^{\prime}$ UTR (Figure 4 and Figure S2). Gene expression is influenced by transcriptional regulation, post-transcriptional regulation, and translational regulation. Since GUS was not detected in p2600 transgenic Arabidopsis, the level of the GUS mRNA was tested. RT-PCRs showed that GUS gene transcripts were present in p2600 transgenic Arabidopsis flowers but not in the corresponding leaf (Figure 6). In other words, GUS gene transcription into mRNA could be specifically driven by the $2600 \mathrm{bp}$ fragment of ZmbZIP25, but this mRNA was not translated correctly. The second intron might not be correctly spliced during the formation of mature mRNA. RT-PCR was performed to test this assumption. The forward primer was in the intronic region, and the reverse primer was in the GUS region. The first intron with a GUS sequence (Intron1+GUS) could not be amplified, while the second intron with a GUS sequence (Intron2+GUS) was successfully amplified (Figure 6). The RT-PCR products were sequenced and confirmed. These results verified that the second intron was still present in the transcripts, which indicated that the inability to drive the expression of the GUS gene with the $2600 \mathrm{bp}$ fragment of ZmbZIP25 in transgenic lines might be due to aberrant pre-mRNA splicing.

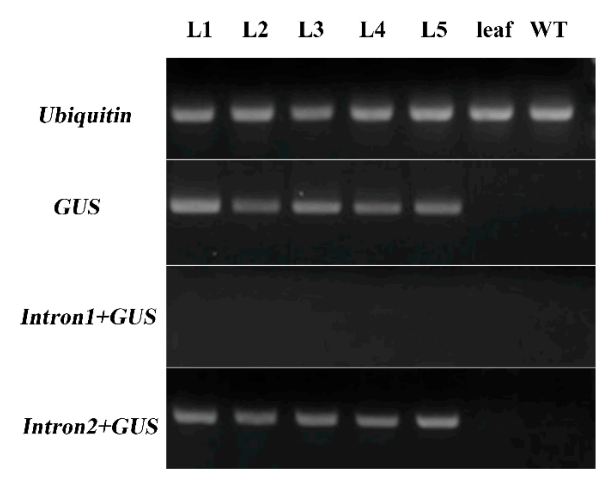

Figure 6. RT-PCR analysis of GUS gene in Arabidopsis. L1-L5 were cDNA templates from flowers of five p2600 transgenic Arabidopsis lines. The two negative control samples were cDNA templates from leaves of p2600 transgenic Arabidopsis and non-transgenic Arabidopsis flowers. Intron1+GUS, the first intron with the GUS sequence with the forward primer in the first intron region and the reverse primer in the GUS region. Intron $2+G U S$, the second intron with the GUS sequence with the forward primer in the second intron region and the reverse primer in the GUS region. Ubiquitin was used as a control.

\subsection{Silk-Specific Transcription in Maize}

To examine the silk-specific expression driven by the ZmbZIP25 promoter in maize, we performed transient expression assays via microprojectile bombardment and Agrobacterium-mediated transformation. After bombarding vectors p2600GFP and p2450GFP into various tissues of maize, we detected GFP (green fluorescent protein) expression. No fluorescence was detected in maize cob, silks, or immature embryos harboring p2600GFP. Maize harboring p2450GFP exhibited green fluorescence in its silks but not in its cob or immature embryos (Figure 7G-L). The vector pCaMV35S-GFP was bombarded to serve as a positive control, resulting in green fluorescence in cob, immature embryos, and silks (Figure 7A-F).

Vectors p2600 and p2450 were also used to probe the expression of the GUS gene driven by the different ZmbZIP25 promoter fragments. Agrobacterium-mediated transformation was performed in maize husk, cob, and silk. Histochemical analysis showed that blue staining was not observed in tissues carrying p2600 (Figure 8E). In p2450-transformed tissues, blue staining was observed in maize silk (Figure 8D) but not in maize husk (Figure 8B), or cob (Figure 8C), or Agrobacterium (Figure 8A), which served as a background control. 


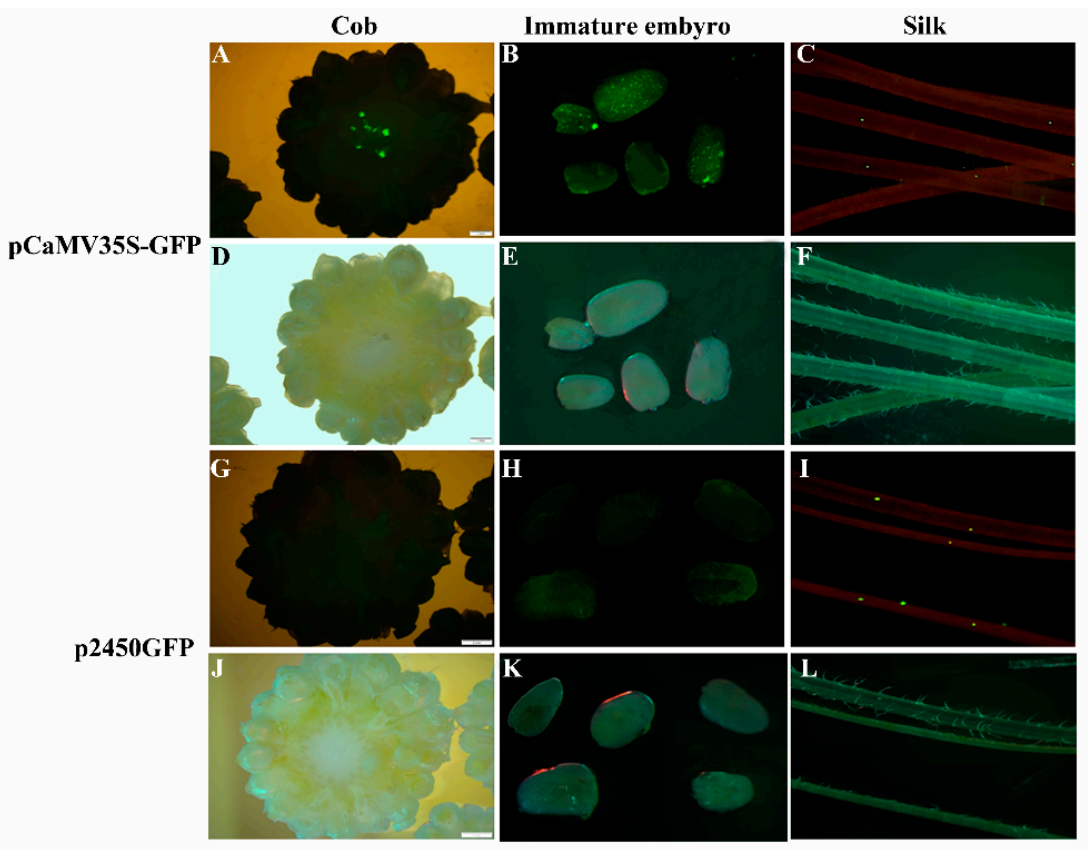

Figure 7. Silk-specific expression driven by the ZmbZIP25 promoter in maize via microprojectile bombardment. Green fluorescence of GFP (green fluorescent protein) expression detected in cob (A), immature embryos (B), and silks (C) bombarded with the vector pCaMV35S-GFP. The corresponding images (D-F) were photographed under light microscopy. Green fluorescence of GFP expression detected in cob (G), immature embryos (H), and silks (I) bombarded with the vector p2450GFP. The corresponding images $(\mathbf{J}-\mathbf{L})$ were photographed under light microscopy. Bars $=2 \mathrm{~mm}$ in $(\mathbf{A}, \mathbf{D}, \mathbf{G}, \mathbf{J})$, and $1 \mathrm{~mm}$ in $(\mathbf{B}, \mathbf{C}, \mathbf{E}, \mathbf{F}, \mathbf{H}, \mathbf{I}, \mathbf{K}, \mathbf{L})$.

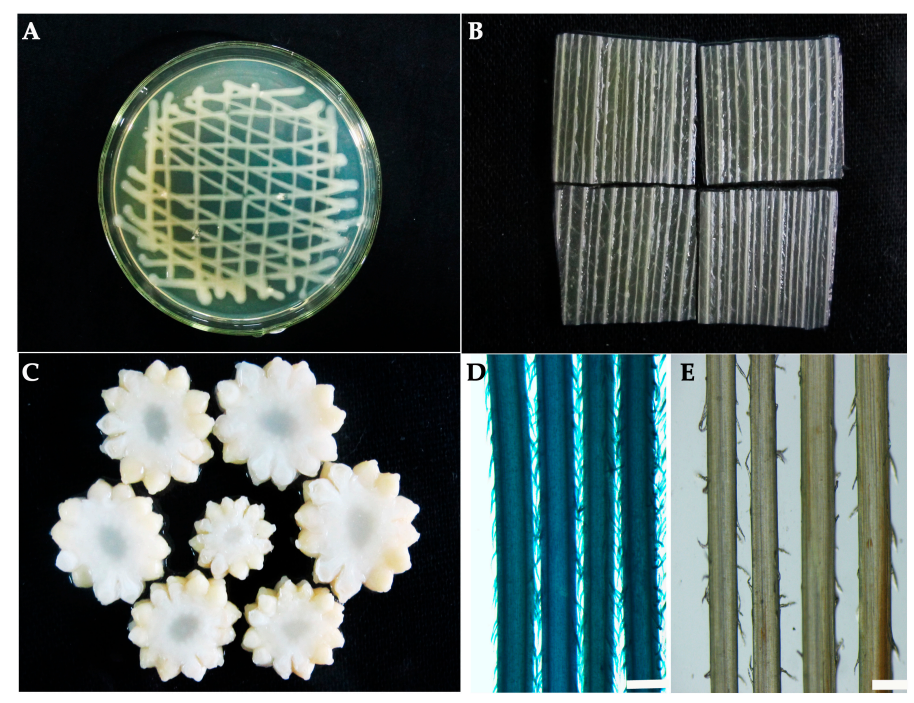

Figure 8. Silk-specific expression driven by the ZmbZIP25 promoter in maize via Agrobacteriummediated transformation. Histochemical GUS analysis of p2450-transformed maize husk (B), cob (C), and silks (D), and Agrobacterium (A), which served as a background. (E) Histochemical GUS analysis of p2600-transformed maize silks. Bars $=500 \mu \mathrm{m}$ in $(\mathbf{D}, \mathbf{E})$.

Transient expression assays in maize consistently showed that the expression of reporter genes was specifically driven by the 2450 bp fragment $(-2083 /+367)$ of ZmbZIP25 and not by the 2600 bp 
fragment $(-2083 /+517)$. The results also verified conclusions drawn from the stable expression assays in Arabidopsis.

\subsection{The -1117 to -957 Region is Responsible for the Silk Specificity of the ZmbZIP25 Promoter}

To determine the specific region involved in ZmbZIP25 gene expression, we generated a series of $5^{\prime}$ deletion fragments (Figure 9A). Five vectors (p1957, p1879, p1695, p1484, and p1324) were constructed and separately transformed into Arabidopsis thaliana col-0. Histochemical analysis indicated that the GUS gene was specifically expressed in Arabidopsis stigmatic papillae harboring p1957 $(-1590 /+367)$ (Figure 9B-D) as well as those of the p1879 $(-1512 /+367)$, p1695 $(-1328 /+367)$, and p1484 $(-1117 /+367)$ transformants. These results implied that the truncated sequences ranging from -1117 to +367 retained full function and specificity. When the fragment was truncated to $1324 \mathrm{bp}$ $(-957 /+367)$, the histochemical assay showed GUS staining in many tissues, including seedling (Figure 9E), rosette leaf (Figure 9F), and whole floral organs (Figure 9G). GUS activity differed in various tissues when p1324 was harbored; this activity was relatively low in seedling root and rosette leaf but relatively high in seedling leaf and the whole flower (Figure 9E-G). This result illustrated that the GUS activity was certainly driven by the region from -957 to +367 but that this region did not encode specificity. The 1324 bp sequence $(-957 /+367)$ was 161 bp shorter than the 1484 bp sequence $(-1117 /+367)$. GUS activity was detected in seedling, rosette leaf, and the whole flower when this $161 \mathrm{bp}$ sequence was lacking, suggesting the possible presence of negative regulatory elements in the region from -1117 to -957 that suppress gene expression in tissues other than stigma.
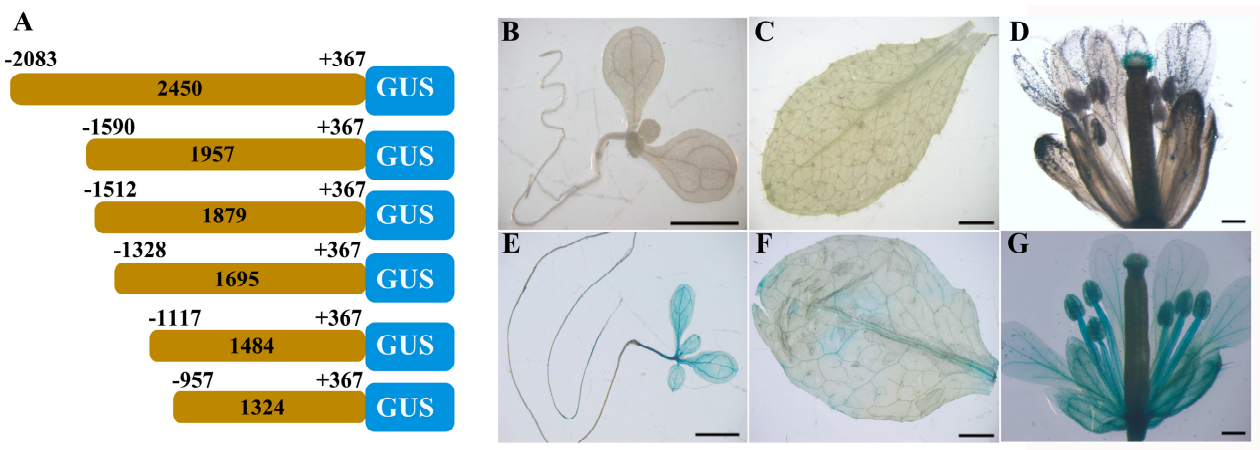

Figure 9. Deletion analysis of ZmbZIP25 5'-flanking sequence. (A) A schematic map of ZmbZIP25 promoter deletion constructs fused to GUS reporter gene that were used for loss-of-function analysis. The gray boxes represent various ZmbZIP25 5'-flanking sequences. The blue boxes represent GUS gene. (B-G) Histochemical staining of GUS gene driven by truncated ZmbZIP25 $5^{\prime}$-flanking fragments in Arabidopsis. ZmbZIP25 5'-flanking fragment $(-1590 /+367)$ in Arabidopsis seedling (B), rosette leaf (C), and flower (D); ZmbZIP25 5'-flanking fragment $(-957 /+367)$ in Arabidopsis seedling (E), rosette leaf $(\mathbf{F})$, and flower $(\mathbf{G})$. At least six independent transformants were examined for each construct. Bars $=500 \mu \mathrm{m}$ in $(\mathbf{D}, \mathbf{G})$, and $2 \mathrm{~mm}$ in $(\mathbf{B}, \mathbf{C}, \mathbf{E}, \mathbf{F})$.

We subsequently analyzed the -1117 to -957 sequence of the ZmbZIP25 promoter. A Box C element (CTCCCAC) was found in the $161 \mathrm{bp}$ region between -1117 and -957 but not in any other positions in the ZmbZIP25 promoter (09/08/2017, http:/ / bioinfo.cau.edu.cn/ProFITS/). The Box C element in the pea asparagine synthetase (AS1) promoter is involved in light-induced transcriptional repression [21]. Box $C$ may be the negative regulatory element causing tissue specificity in maize silks of ZmbZIP25.

\section{Discussion}

ZmbZIP25 contains a bZIP domain and a DOG1 domain and belongs to the D group of the 11 groups of the maize bZIP proteins according to its phylogenetic relationship $[17,22]$. Genes in group 
D might participate in the pathogen response [17]. TGA6, which belongs to the TGA family in bZIP group D, is speculated to function in defense against pathogens in Arabidopsis [23]. Bioinformatic predictions indicate that some genes in group $\mathrm{D}$ that show similar patterns of expression are involved with fungal infections [17]. TGA2 and TGA3 interact with NPR1, bind SA-responsive elements in the Arabidopsis pathogenesis-related (PR-1) promoter, and are involved in disease resistance pathways [24]. ZmbZIP25 may regulate the expression of pathogen resistance genes in maize silk by interacting with other factors. The exact function and regulation of ZmbZIP25 are being investigated.

bZIP transcription factors may regulate cell-type-specific gene expression in plants. Grp1.8 (glycine-rich protein) exhibited xylem-specific expression in tomato. bZIP transcriptional activator VSF-1 bound a 28 bp element in the grp1.8 promoter (vs-1) to regulate grp1.8 expression [25]. Transcriptome data and RT-PCR results showed that ZmbZIP25 had high-level expression in silks and was barely detected in other tissues (Figure 1). In situ hybridization assays indicated that ZmbZIP25 was expressed exclusively in the xylem of maize silk cells (Figure 2). Few genes have been reported that are expressed specifically in the xylem of maize silk. Our research on ZmbZIP25 enriches the study of xylem-specific promoters.

A $5^{\prime}$ RACE assay was performed to identify the TSS of ZmbZIP25 (Figure 3). However, no TATA box was present within 50 bp upstream of the TSS. Two YTCANTYY initiator elements were found in the ZmbZIP25 promoter (http:/ / bioinfo.cau.edu.cn/ProFITS/) (Figures 4 and S2). In some TATA-less promoters, pyrimidine-rich initiator (Inr) elements that overlap the TSS compensate for the lack of a TATA box and direct basal transcription initiation [26-28]. The tobacco photosystem I $p s a D b$ gene promoter responds to light and depends on an Inr but not a TATA box [29]. The promoter of $P i b$, a member of the NBS-LRR (nucleotide-binding site leucine-rich repeat) class of plant disease resistance genes, contains six YTCANTYY motifs and makes the expression of $P i b$ in darkness specific to root $[30,31]$. The ZmbZIP25 promoter is expressed exclusively in silk and may be associated with pathogen resistance based on the YTCANTYY motif. Some pathogen-responsive elements, such as the AGCCGCC motif and the GCC-box, are also present in the ZmbZIP25 promoter. The precise mechanism by which ZmbZIP25 participates in pathogen resistance remains unknown, but further research is underway.

Considering that the stable expression assay in maize plants requires a long period of time and that its low transformation efficiency makes it difficult to realize, the model plant Arabidopsis was selected as an alternative system due to its relatively short life cycle and highly efficient transformation [32]. The histochemical analysis of GUS genes showed that blue staining occurred in Arabidopsis stigma papillae only when harboring p2450 (and not when harboring p2600, Figure 5). These results were also confirmed via transient expression assays in maize (Figures 7 and 8).

The difference between the 2450 and $2600 \mathrm{bp}$ fragments was a deletion of $151 \mathrm{bp}$ from the $3^{\prime}$ end. This 151 bp region contains part of the second intron and part of the $5^{\prime}$ UTR (Figure 4 and Figure S2). RT-PCRs verified that the second intron was still present in transcripts (Figure 6). The precise removal of pre-mRNA introns is critical for gene expression [33]. The conformation of the pre-mRNA influences the accessibility of splice sites. The number of structural options increases with the molecule's length. The secondary structures of GUS pre-mRNA in the p2600 transgenic line may differ from those in the p2450 transgenic line, affecting pre-mRNA splicing. A splicing error that adds or removes even $1 \mathrm{nt}$ will perturb the open reading frame of an mRNA [34]. Aberrant pre-mRNA splicing of the second intron of p2600 might lead to the inability to express the GUS gene in transgenic Arabidopsis.

A gene that displays specific expression may be influenced by its regulatory elements, including negative elements and interacting transcriptional factors. In plants, the sequence CGTG(T/C)G of the $C P D$ (constitutive photomorphogenesis and dwarfism gene) promoter was tested as a binding site for transcription repressor BZR1 to regulate brassinosteroid signaling and downstream growth responses [35]. In Arabidopsis, FRS7 and FRS12 affect flowering time and growth partly by binding to the promoters and repressing the expression of GIGANTEA and PHYTOCHROME INTERACTING FACTOR 4 as well as several of their downstream signaling targets [36]. A series of deletion analyses showed 
that a reporter gene driven by the truncated 1324 bp region $(-957 /+367)$ in ZmbZIP25 was expressed not only in the stigmatic papillae but also in other tissues (Figure 9). This result implied the possible presence of negative cis-elements in the region of ZmbZIP25 from - 1117 to -957 that can suppress gene expression in tissues other than silk. A Box C element (CTCCCAC) was found only within the $161 \mathrm{bp}$ region from -1117 to -957 (09/08/2017, http:/ /bioinfo.cau.edu.cn/ProFITS/), which was identified as a cis-element in the pea asparagine synthetase (AS1) promoter that participated in light-induced transcriptional repression [21]. We speculate that Box $C$ tends to be a negative regulatory element in ZmbZIP25 and functions in a tissue-specific manner in maize silks. To confirm the negative regulatory cis-element sequences in the region from -1117 to -957 of ZmbZIP25, further studies are required, such as those involving the creation of point mutations in the promoter and the examination of DNA-protein binding and protein-protein interactions.

In conclusion, our findings suggest that the region from -1117 to -957 of the ZmbZIP25 $5^{\prime}$-flanking sequence is necessary for silk-specific gene expression. The second intron of ZmbZIP25, involved in aberrant pre-mRNA splicing, may provide insight into tissue-specific promoter research. The cis-elements and mechanism of ZmbZIP25 silk-specific expression should be further explored. Moreover, the ZmbZIP25 5'-flanking sequence, as a strong silk-specific promoter, has potential agricultural application value in areas such as female sterility.

\section{Materials and Methods}

\subsection{RT-PCR Analysis}

Total RNA was isolated from various tissues of maize (Zea mays L. cv Mo17) using an RNAprep Pure Plant Kit (Tiangen, Dalian, China) according to the manufacturer's protocol. The first-strand cDNA was synthesized with AMV (avian myeloblastosis virus) reverse transcriptase (Promega, Madison, WI, USA). The ZmbZIP25 transcription products were amplified using the specific primers RT-ZIP-F (5'-CGACCAGCAGCCAAACTCTA-3') and RT-ZIP-R (5'-AATCCGCCCAGCCACATAAA-3'). The maize actin gene was used as a reference control. This amplification experiment was repeated three times.

A similar procedure was performed in Arabidopsis. GUS transcription products were amplified using the specific primers GUS-F (5'-CGACTGGGCAGATGAACATG-3') and GUS-R (5'-TACTCCACATCACCACGCTT-3'). The transcription product containing the first intron and GUS (Intron $1+$ GUS) was amplified using the specific primers F1 (5'-TGCTCCAGGCAACCTTGTTT-3') and R1 (5'-TCCACAGTTTTCGCGATCCA-3'). The transcription product containing the second intron and GUS (Intron2+GUS) was amplified using the specific primers F2 (5'-CCGGTGAGCCGATGATTTCT-3') and R2 (5'-CCTGCCCAACCTTTCGGTAT-3'). The Arabidopsis ubiquitin gene was used as a reference control.

\subsection{In Situ RNA Hybridization}

Fresh silks were collected from maize plants on the day of emergence, cut into 2-3 mm lengths, and then immediately fixed in FAA solution (50\% ethanol, 5\% formaldehyde, and 5\% acetic acid). The gene-specific region of ZmbZIP25 (+631 to +1035, counted from the ATG start codon) was amplified as a template for synthesizing sense and antisense RNA probes. The probes were labeled with digoxigenin using a DIG Northern Starter Kit (Roche Diagnostics, Mannheim, Germany). The silks were dehydrated, embedded in paraffin (Sigma-Aldrich, Saint Louis, MO, USA), sliced to an $8 \mu \mathrm{m}$ thickness, pretreated, hybridized, washed, and detected as previously described [37].

\subsection{Identification of ZmbZIP25 Gene Transcription Start Site}

To identify the TSS of the ZmbZIP25 gene, 5' RACE was performed using a FirstChoice ${ }^{\circledR}$ RLM-RACE Kit (Ambion, Vilnius, Lithuania). Total RNA was treated with calf intestinal phosphatase (CIP) and then decapped with tobacco acid pyrophosphatase (TAP). A 45 base RNA adapter 
oligonucleotide was ligated to the RNA population using T4 RNA ligase. A random-primed reverse transcription reaction and nested PCR were then used to amplify the $5^{\prime}$ end of a specific transcript. Two gene-specific antisense primers (outer-specific primer: $5^{\prime}$-AGTAGAGTTTGGCTGCTGGT-3' and inner-specific primer: $5^{\prime}$-CCTTCTTTCTTAGACGGCTTTT-3') were designed to amplify with the sense primers provided with the kit. The amplified PCR products were sequenced.

\subsection{Construction of Expression Vectors}

The 5'-flanking sequences of ZmbZIP25 were amplified by PCR, and a $2600 \mathrm{bp}$ fragment $(-2083 /+517)$ and a $2450 \mathrm{bp}$ fragment $(-2083 /+367)$ were used to construct vectors. We used a pCaMV35S-GFP backbone and the above fragments to construct p2600GFP and p2450GFP, respectively, which no longer had the CaMV35S promoter to drive the expression of the GFP reporter gene. pCaMV35S-GFP, which served as a control, was modified from vector pUC-SPYCE by replacing YFPC $^{C}$ with GFP [38].

Seven different promoter fragments $(-2083 /+517,-2083 /+367,-1590 /+367,-1512 /+367$, $-1328 /+367,-1117 /+367$, and $-957 /+367)$ were cloned. These fragments were inserted into the binary vector pCAMBIA1391 at its EcoRI and SpeI sites to drive GUS gene expression. The created vectors were named p2600, p2450, p1957, p1879, p1695, p1484, and p1324, respectively.

\subsection{Microprojectile Bombardment and Transient Expression Assay in Maize}

Maize plants were grown in soil in a controlled-environment greenhouse $\left(30^{\circ} \mathrm{C}, 14 \mathrm{~h}\right.$ light $/ 20^{\circ} \mathrm{C}$, $10 \mathrm{~h}$ dark). The cobs and silks were obtained separately from fresh non-pollinated maize ears. Cobs were cut into 5-mm-thick cross sections. Silks were cut into approximately $2.5 \mathrm{~cm}$ lengths. The immature embryos were peeled $15 \mathrm{~d}$ after pollination. All the tissues were pretreated with hypertonic medium ( $0.4 \mathrm{M}$ mannitol in NB Basal medium) for $4 \mathrm{~h}$ before bombardment. A biolistic PDS-1000/He system (Bio-Rad, Hercules, CA, USA) was used to bombard the plasmid DNA p2600GFP, p2450GFP, and pCaMV35S-GFP, carried by gold particles, under 1300 psi of helium. After bombardment, the tissues were maintained in the hypertonic medium overnight. GFP fluorescence was observed under an Olympus fluorescence microscope (SZX-16, Tokyo, Japan).

\subsection{Agrobacterium-Mediated Transformation and Transient Expression Analysis}

Agrobacterium-mediated transformation was performed according to the method of Frame et al. [39] with some modifications. Various tissues of maize, including husk, cob, and silk, were freshly harvested from greenhouse-grown maize plants and infected with an Agrobacterium strain GV3101 (harboring plasmid p2600 or p2450) suspension $\left(\mathrm{OD}_{600}=0.3-0.4\right)$ for $30 \mathrm{~min}$. After infection, all the tissues were dried on filter paper, transferred to co-cultivation medium, and then incubated in the dark at $20{ }^{\circ} \mathrm{C}$ for 2 days. Before they were stained, the tissues were washed with $\mathrm{ddH}_{2} \mathrm{O}$ to remove the Agrobacterium cells from the tissue surfaces. Histochemical analysis was performed to test the GUS activity.

\subsection{Arabidopsis thaliana Transformation and Selection}

Arabidopsis thaliana Columbia-0 was transformed with Agrobacterium tumefaciens strain GV3101 cells harboring a plasmid (p2600, p2450, p1957, p1879, p1695, p1484, or p1324) using the floral dip method [40,41]. Transformed seeds were germinated on MS medium (Murashige and Skoog medium) containing $50 \mathrm{mg} / \mathrm{L}$ hygromycin for selection. Resistant transgenic Arabidopsis plants were confirmed by PCR with the primers bZIP25-F (CGACCAGCAGCCAAACTCTA) and bZIP25-R (AATCCGCCCAGCCACATAAA). More than five independent lines of the $\mathrm{T}_{2}$ generation were used for further analysis. 


\subsection{GUS Activity Assays}

Histochemical assays for GUS activity were performed as described by Lang et al. [42], with modification. The GUS reaction buffer contained $100 \mathrm{mM}$ sodium phosphate buffer ( $\mathrm{pH} 7.0)$ with $1 \mathrm{mg} / \mathrm{mL}$ X-Gluc (5-bromo-4-chloro-3-indolyl- $\beta$-D-glucuronide) as the substrate with $5 \mathrm{mM}$ $\mathrm{K}_{3} \mathrm{Fe}(\mathrm{CN})_{6}, 5 \mathrm{mM} \mathrm{K}_{4} \mathrm{Fe}(\mathrm{CN})_{6}, 0.5 \mathrm{mM}$ EDTA (Ethylenediaminetetraacetic acid), $0.1 \%$ Triton X-100, and $20 \%$ methanol. Samples were vacuum-filtered and then incubated in GUS reaction buffer at $37^{\circ} \mathrm{C}$ overnight in the dark. Then, $70 \%$ ethanol was used for decoloration. The samples were photographed under a stereo microscope (Olympus SEX16, Tokyo, Japan).

Supplementary Materials: The following are available online at www.mdpi.com/1422-0067/19/3/822/s1.

Acknowledgments: This work was supported by the projects (No. 2016ZX08009003-004) from the Ministry of Agriculture of China for Transgenic Research and National Natural Science Foundation (Grant No. 31171258).

Author Contributions: Qian Zhao and Wanying Li conceived and designed the research; Wanying Li prepared the samples, designed and performed the experiments, and wrote the original manuscript; Dan Yu contributed to the lab experiment; Jingjuan Yu assisted in research and manuscript discussion; Dengyun Zhu helped in maize planting; Qian Zhao thoroughly revised and finalized the manuscript. All authors read and approved the final manuscript.

Conflicts of Interest: The authors declare no conflict of interest.

\section{Abbreviations}

$\begin{array}{ll}\text { bZIP } & \text { basic leucine zipper } \\ \text { DOG1 } & \text { delay of germination 1 } \\ \text { PR } & \text { pathogenesis-related } \\ 5^{\prime} \text {-UTR } & 5^{\prime} \text {-untranslated region }\end{array}$

\section{References}

1. Heslop-Harrison, Y.; Reger, B.J.; Heslop-Harrison, J. The pollen-stigma interaction in the grasses. 5. Tissue organisation and cytochemistry of the stigma ("silk") of Zea mays L. Acta Bot. Neerl. 1984, 33, 81-99. [CrossRef]

2. Miller, E.C. Development of the pistillate spikelet and fertilization in Zea mays L. J. Agric. Res. 1919, 18, 255-265.

3. Kroh, M.; Gorissen, M.H.; Pfahler, P.L. Ultrastructural studies on styles and pollen tubes of Zea mays L. General survey on pollen tube growth in vivo. Acta Bot. Neerl. 1979, 28, 513-518. [CrossRef]

4. Xu, X.H.; Chen, H.; Sang, Y.L.; Wang, F.; Ma, J.P.; Gao, X.Q.; Zhang, X.S. Identification of genes specifically or preferentially expressed in maize silk reveals similarity and diversity in transcript abundance of different dry stigmas. BMC Genom. 2012, 13, 294. [CrossRef] [PubMed]

5. McInnis, S.M.; Costa, L.M.; Gutiérrez-Marcos, J.F.; Henderson, C.A.; Hiscock, S.J. Isolation and characterization of a polymorphic stigma-specific class III peroxidase gene from Senecio squalidus L. (Asteraceae). Plant Mol. Biol. 2005, 57, 659-677. [CrossRef] [PubMed]

6. McInnis, S.M.; Emery, D.C.; Porter, R.; Desikan, R.; Hancock, J.T.; Hiscock, S.J. The role of stigma peroxidases in flowering plants: Insights from further characterization of a stigma-specific peroxidase (SSP) from Senecio squalidus (Asteraceae). J. Exp. Bot. 2006, 57, 1835-1846. [CrossRef] [PubMed]

7. Stein, J.C.; Dixit, R.; Nasrallah, M.E.; Nasrallah, J.B. SRK, the stigma-specific $S$ locus receptor kinase of Brassica, is targeted to the plasma membrane in transgenic tobacco. Plant Cell 1996, 8, 429-445. [CrossRef] [PubMed]

8. Atkinson, A.H.; Heath, R.L.; Simpson, R.J.; Clarke, A.E.; Anderson, M.A. Proteinase inhibitors in Nicotiana alata stigmas are derived from a precursor protein which is processed into five homologous inhibitors. Plant Cell 1993, 5, 203-213. [CrossRef] [PubMed]

9. Trick, M.; Heizmann, P. Sporophytic self-incompatibility systems: Brassica S gene family. Int. Rev. Cytol. 1992, 140, 485-524. [PubMed] 
10. Dzelzkalns, V.A.; Thorsness, M.K.; Dwyer, K.G.; Baxter, J.S.; Balent, M.A.; Nasrallah, M.E.; Nasrallah, J.B. Distinct cis-acting elements direct pistil-specific and pollen-specific activity of the Brassica $S$ locus glycoprotein gene promoter. Plant Cell 1993, 5, 855-863. [CrossRef] [PubMed]

11. Goring, D.R.; Glavin, T.L.; Schafer, U.; Rothstein, S.J. An $S$ receptor kinase gene in self-compatible Brassica napus has a 1-bp deletion. Plant Cell 1993, 5, 531-539. [CrossRef] [PubMed]

12. Nasrallah, J.B.; Nasrallah, M.E. Pollen-stigma signaling in the sporophytic self-incompatibility response. Plant Cell 1993, 5, 1325-1335. [CrossRef] [PubMed]

13. Tao, T.Y.; Ouellet, T.; Dadej, K.; Miller, S.S.; Johnson, D.A.; Singh, J. Characterization of a novel glycine-rich protein from the cell wall of maize silk tissues. Plant Cell Rep. 2006, 25, 848-858. [CrossRef] [PubMed]

14. Reid, L.M.; Bolton, A.T.; Hamilton, R.I.; Woldemariam, T. Effect of silk age on resistance of maize to Fusarium graminearum. Can. J. Plant Pathol. 1992, 14, 293-298. [CrossRef]

15. Hazzard, R.V.; Schultz, B.B.; Groden, E.; Ngollo, E.D.; Seidlecki, E. Evaluation of oils and microbial pathogens for control of Lepidopteran pests of sweet corn New England. J. Econ. Entomol. 2003, 96, 1653-1661. [CrossRef] [PubMed]

16. Johnson, E.T.; Berhow, M.A.; Dowd, P.F. Expression of a maize Myb transcription factor driven by a putative silk-specific promoter significantly enhances resistance to Helicoverpa zea in transgenic maize. J. Agric. Food Chem. 2007, 55, 2998-3003. [CrossRef] [PubMed]

17. Wei, K.; Chen, J.; Wang, Y.; Chen, Y.; Chen, S.; Lin, Y.; Pan, S.; Zhong, X.; Xie, D. Genome-wide analysis of bZIP-encoding genes in maize. DNA Res. 2012, 19, 463-476. [CrossRef] [PubMed]

18. Nakabayashi, K.; Bartsch, M.; Xiang, Y.; Miatton, E.; Pellengahr, S.; Yano, R.; Seo, M.; Soppe, W.J. The time required for dormancy release in Arabidopsis is determined by DELAY OF GERMINATION1 protein levels in freshly harvested seeds. Plant Cell 2012, 24, 2826-2838. [CrossRef] [PubMed]

19. Joshi, C.P. An inspection of the domain between putative TATA box and translation start site in 79 plant genes. Nucleic Acids Res. 1987, 15, 6643-6653. [CrossRef] [PubMed]

20. Tanaka, M.; Takano, J.; Chiba, Y.; Lombardo, F.; Ogasawara, Y.; Onouchi, H.; Naito, S.; Fujiwara, T. Boron-dependent degradation of NIP5; 1 mRNA for acclimation to excess boron conditions in Arabidopsis. Plant Cell 2011, 23, 3547-3559. [CrossRef] [PubMed]

21. Ngai, N.; Tsai, F.Y.; Coruzzi, G. Light-induced transcriptional repression of the pea AS1 gene: Identification of cis-elements and transfactors. Plant J. 1997, 12, 1021-1034. [CrossRef] [PubMed]

22. Jakoby, M.; Weisshaar, B.; Dröge-Laser, W.; Vicente-Carbajosa, J.; Tiedemann, J.; Kroj, T.; Parcy, F. bZIP transcription factors in Arabidopsis. Trends Plant Sci. 2002, 7, 106-111. [CrossRef]

23. Xiang, C.; Miao, Z.; Lam, E. DNA-binding properties, genomic organization and expression pattern of TGA6, a new member of the TGA family of bZIP transcription factors in Arabidopsis thaliana. Plant Mol. Biol. 1997, 34, 403-415. [CrossRef] [PubMed]

24. Zhou, J.M.; Trifa, Y.; Silva, H.; Pontier, D.; Lam, E.; Shah, J.; Klessig, D.F. NPR1 differentially interacts with members of the TGA/OBF family of transcription factors that bind an element of the $P R-1$ gene required for induction by salicylic acid. Mol. Plant Microbe Interact. 2000, 13, 191-202. [CrossRef] [PubMed]

25. Torres-Schumann, S.; Ringli, C.; Heierli, D.; Amrhein, N.; Keller, B. In vitro binding of the tomato bZIP transcriptional activator VSF-1 to a regulatory element that controls xylem-specific gene expression. Plant J. 1996, 9, 283-296. [CrossRef] [PubMed]

26. Smale, S.T.; Baltimore, D. The "Initiator" as a transcription control element. Cell 1989, 57, 103-113. [CrossRef]

27. Smale, S.T.; Jain, A.; Kaufmann, J.; Emami, K.H.; Lo, K.; Garraway, I.P. The initiator element: A paradigm for core promoter heterogeneity within metazoan protein-coding genes. Cold Spring Harb. Symp. Quant. Biol. 1998, 63, 21-31. [CrossRef] [PubMed]

28. Smale, S.T. Transcription initiation from TATA-less promoters within eukaryotic protein-coding genes. Biochim. Biophys. Acta 1997, 1351, 73-88. [CrossRef]

29. Nakamura, M.; Tsunoda, T.; Obokata, J. Photosynthesis nuclear genes generally lack TATA-boxes: A tobacco photosystem I gene responds to light through an initiator. Plant J. 2002, 29, 1-10. [CrossRef] [PubMed]

30. Wang, Z.X.; Yano, M.; Yamanouchi, U.; Iwamoto, M.; Monna, L.; Hayasaka, H.; Katayose, Y.; Sasaki, T. The Pib gene for rice blast resistance belongs to the nucleotide binding and leucine-rich repeat class of plant disease resistance genes. Plant J. 1999, 19, 55-64. [CrossRef] [PubMed]

31. Shao, K.Q.; Yang, S.H.; Yu, L.; Wan, J.M. Function verifying of darkness inducing motif YTCANTYY in Pib promoter via rice transformation. Acta Agron. Sin. 2008, 9, 28. [CrossRef] 
32. Meinke, D.W.; Cherry, J.M.; Dean, C.; Rounsley, S.D.; Koornneef, M. Arabidopsis thaliana: A model plant for genome analysis. Science 1998, 282, 662-682. [CrossRef] [PubMed]

33. Hastings, M.L.; Krainer, A.R. Pre-mRNA splicing in the new millennium. Curr. Opin. Cell Biol. 2001, 13, 302-309. [CrossRef]

34. Faustino, N.A.; Cooper, T.A. Pre-mRNA splicing and human disease. Genes Dev. 2003, 17, 419-437. [CrossRef] [PubMed]

35. He, J.X.; Gendrn, J.M.; Sun, Y.; Gampala, S.S.L.; Gendron, N.; Sun, C.Q.; Wang, Z.Y. BZR1 is a transcriptional repressor with dual roles in brassinosteroid homeostasis and growth responses. Science 2005, 307, 1364-1368. [CrossRef] [PubMed]

36. Ritter, A.; Inigo, S.; Fernández-Calvo, P.; Heyndrickx, K.S.; Dhondt, S.; Shi, H.; De Milde, L.; Vanden Bossche, R.; De Clercq, R.; Eeckhout, D.; et al. The transcriptional repressor complex FRS7-FRS12 regulates flowering time and growth in Arabidopsis. Nat. Commun. 2017, 8, 15235. [CrossRef] [PubMed]

37. Kouchi, H.; Hata, S. Isolation and characterization of novel nodulin cDNAs representing genes expressed at early stages of soybean nodule development. Mol. Genom. Genet. 1993, 238, 106-119.

38. Walter, M.; Chaban, C.; Schütze, K.; Batistic, O.; Weckermann, K.; Näke, C.; Blazevic, D.; Grefen, C.; Schumacher, K.; Oecking, C.; et al. Visualization of protein interactions in living plant cells using bimolecular fluorescence complementation. Plant J. 2004, 40, 428-438. [CrossRef] [PubMed]

39. Frame, B.R.; Shou, H.; Chikwamba, R.K.; Zhang, Z.; Xiang, C.; Fonger, T.M.; Pegg, S.E.; Li, B.; Nettleton, D.S.; Pei, D.; Wang, K. Agrobacterium tumefaciens-mediated transformation of maize embryos using a standard binary vector system. Plant Physiol. 2002, 129, 13-22. [CrossRef] [PubMed]

40. Clough, S.J.; Bent, A.F. Floral dip: A simplified method for Agrobacterium-mediated transformation of Arabidopsis thaliana. Plant J. 1998, 16, 735-743. [CrossRef] [PubMed]

41. Weigel, R.; Glazebrook, J. Arabidopsis: A Laboratory Manual; Cold Spring Harbor Laboratory Press: Cold Spring Harbor, NY, USA, 2002.

42. Lang, Z.; Zhou, P.; Yu, J.; Ao, G.; Zhao, Q. Functional characterization of the pollen-specific $S B g L R$ promoter from potato (Solanum tuberosum L.). Planta 2008, 227, 387-396. [CrossRef] [PubMed]

(C) 2018 by the authors. Licensee MDPI, Basel, Switzerland. This article is an open access article distributed under the terms and conditions of the Creative Commons Attribution (CC BY) license (http:/ / creativecommons.org/licenses/by/4.0/). 